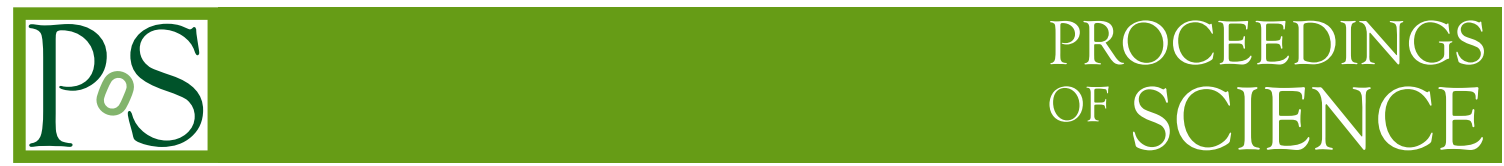

\title{
QCD with Weak Bosons and Jets with ATLAS
}

\author{
Guillaume Kirsch*on behalf of the ATLAS Collaboration \\ University of Oxford, Oxford \\ E-mail: guillaume.kirsch@cern.ch,kristin.lohwasser@cern.ch
}

\begin{abstract}
The study of $W$ and $Z$ boson production in association with jets in $p p$ collisions at $14 \mathrm{TeV}$ is an important element of the physics program within ATLAS. These processes are not only interesting in their own right as tests of perturbative quantum chromodynamics (QCD) at the LHC, but also constitute important backgrounds for both Standard Model and Beyond Standard Model physics processes. In these proceedings the benefits and theoretical motivations for the analysis of $W / Z+$ jets events are presented and the experimental challenges briefly reviewed.
\end{abstract}

Physics at LHC 2008

29 September - October 4, 2008

Split, Croatia

\footnotetext{
*Speaker.

${ }^{\dagger}$ Prepared the poster. Kristin Lohwasser (supported by DAAD) presented the poster and wrote these proceedings.
} 

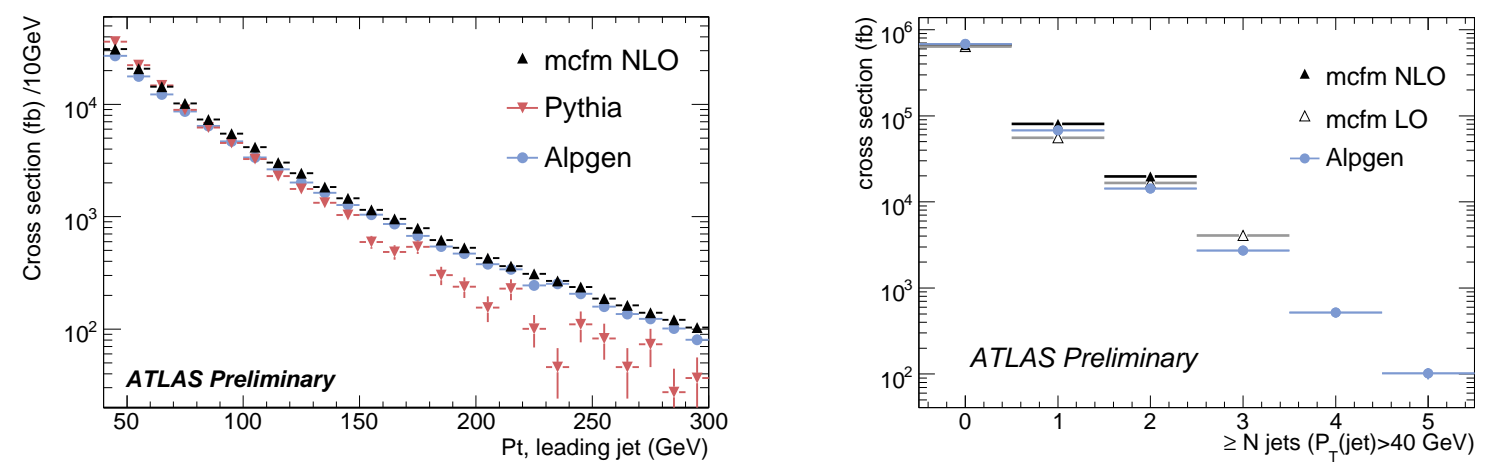

Figure 1: The left plot shows a comparison of the leading jet $p_{T}$ for $Z \rightarrow \mu \mu+$ jets samples obtained using ALPGEN, PYTHIA and NLO calculations from MCFM. On the right hand side a comparison for the predictions for jet multiplicities using ALPGEN and the NLO as well as LO MCFM calculation for two and three additional parton legs is depicted for a $Z \rightarrow e e+$ jets sample. For ALPGEN and PYTHIA the statistical uncertainties are shown and for MCFM the systematic uncertainties from unfolding to the hadron level and the PDFs are shown.

\section{Theoretical motivations}

The study of $W$ and $Z$ boson production in association with jets is a refinement of early electroweak measurements that will be undertaken with the ATLAS detector. In order to predict these processes one relies heavily on pertubative QCD to describe the hard scatter and radiative corrections that represent additional parton legs to the boson production. Also, $W / Z+j e t s$ are expected to be one of the dominant backgrounds to various physics beyond the Standard Model, making up to $10 \%$ of the background in searches using a signature with one lepton, missing energy and jets [1].

Monte-Carlo studies investigated $Z \rightarrow \mu \mu+$ jets samples generated with ALPGEN, PYTHIA and NLO analytic calculations from MCFM using an integrated luminosity of $1 \mathrm{fb}^{-1}$ (for details [1]). The comparison of the leading jet $p_{T}$, where the jets in question were obtained from generator level hadrons for the generators and from partons using hadronization corrections for the calculations, show discrepancies between the predictions (fig. 1, left). While ALPGEN agrees with the MCFM NLO calculation, PYTHIA predicts a much softer jet $p_{T}$ spectrum. This is expected since additional jets in PYTHIA arise from the soft parton shower and not from additional hard partons. The predictions for the cross sections for $Z \rightarrow e e+j e t s$ events as a function of jet multiplicity for jets above $40 \mathrm{GeV}$ are compared on the right hand side of fig. 1. Here, the NLO as well as LO MCFM calculations for two and three parton legs, i.e. two or three jets in the event, are compared to the ALPGEN event generator. While the various predictions agree perfectly for the 0 jet case, discrepancies of about $15 \%$ show up for higher multiplicities. This emphasizes the need to validate the predictions by experiment. 


\section{Experimental challenges}

Analyses of $W / Z+$ jets events face additional difficulties: systematic errors arise not only from the selection of the electroweak boson, but also from the reconstruction of the jets. The reconstruction of leptons and of $B_{T}$ becomes more complex in the presence of a multi-jet final state. In particular, the distances between leptons and jets decrease with jet multiplicity.

Examples of these experimental challenges are shown in fig.2, where on the left hand side the single lepton efficiency is depicted as a function of jet multiplicity. Here, efficiencies obtained from simulated $Z \rightarrow e e$ data using a data-driven tag-and-probe method are shown as markers. For comparison the efficiencies calculated using the Monte Carlo truth information are drawn as error bands. Increased jet activity decreases the efficiency to trigger events, due to a veto cut on hadronic energy close to the lepton trigger. The systematic error in the determination of the efficiency increases as the agreement between MC truth and data-driven estimate deteriorates, since the tagand-probe method selects slightly cleaner events. Another systematic uncertainty arises from the jet energy scale (JES). This effect is shown on the right hand side of fig.2, where the error on the measured cross section of $Z \rightarrow \mu \mu+$ jets events is depicted as function of jet multiplicity. JES uncertainties of 1,5 and $10 \%$ lead to errors on the cross section of up to $40 \%$.

\section{Outlook}

While the measurement of $W / Z+$ jets is experimentally challenging, the results are theoretically relevant and a vital ingredient of the ATLAS physics programme. The primary result of the analyses with real data will be the determination of cross sections as function of various jet parameters.

\section{References}

[1] ATLAS Collaboration, Expected Performance of the ATLAS Experiment, Detector, Trigger and Physics, 2008, [CERN-OPEN-2008-020]
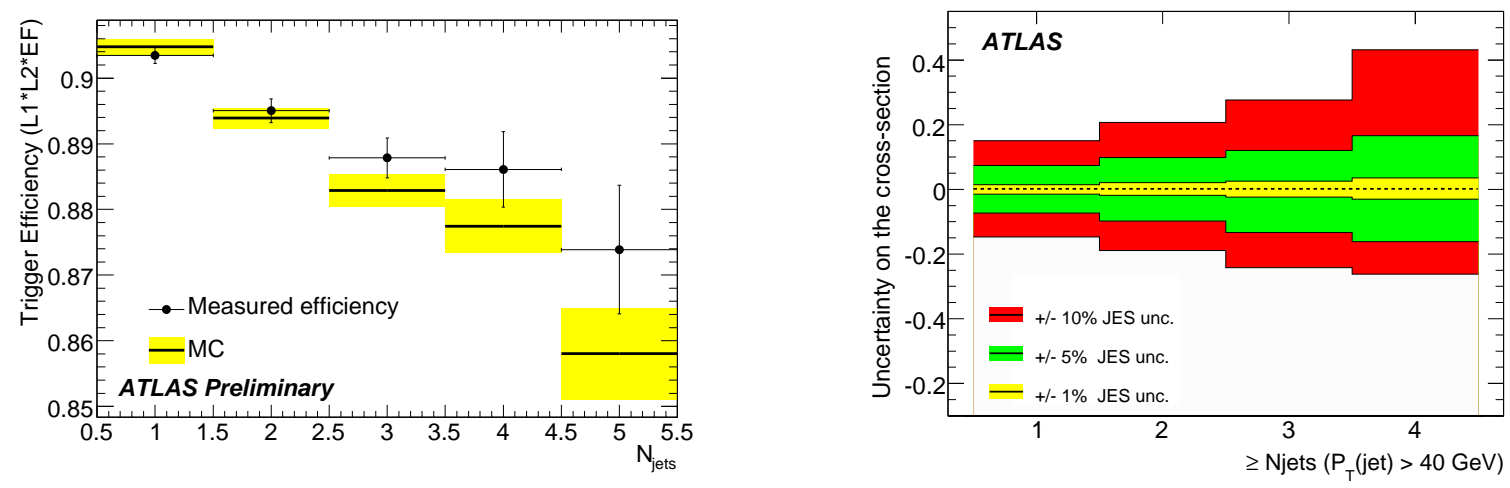

Figure 2: The left plot shows the single electron efficiency as a function of jet multiplicity of jets above $40 \mathrm{GeV}$ for efficiencies derived using the tag-and-probe method with a $Z \rightarrow e e$ sample (data points) and using Monte Carlo information (error bands). The right plot shows the systematic error arising from the uncertainty on the JES on the cross section of $Z \rightarrow \mu \mu+$ jets events as function of jet multiplicity. 